\title{
Impact of synovial fluid flow on temperature regulation in knee cartilage
}

\author{
Mohamadreza Nassajian Moghadam, Philippe Abdel-Sayed, \\ Valérie Malfroy Camine, Dominique P. Pioletti* \\ Laboratory of Biomechanical Orthopedics, Institute of Bioengineering, École Polytechnique Fédérale de Lausanne (EPFL), Lausanne, Switzerland
}

\section{A R T I C L E I N F O}

\section{Article history:}

Accepted 5 November 2014

\section{Keywords:}

Cartilage

Dissipation

Synovial fluid

Poroelasticity

Heat

\begin{abstract}
A B S T R A C T
Several studies have reported an increase of temperature in cartilage submitted to cyclic sinusoidal loading. The temperature increase is in part due to the viscous behavior of this tissue, which partially dissipates the input mechanical energy into heat. While the synovial fluid flow within the intra-articular gap and inside the porous cartilage is supposed to play an important role in the regulation of the cartilage temperature, no specific study has evaluated this aspect. In the present numerical study, a poroelastic model of the knee cartilage is developed to evaluate first the temperature increase in the cartilage due to dissipation and second the impact of the synovial fluid flow in the cartilage heat transfer phenomenon. Our results showed that, the local temperature is effectively increased in knee cartilage due to its viscous behavior. The synovial fluid flow cannot significantly preventing this phenomenon. We explain this result by the low permeability of cartilage and the moderate fluid exchange at the surface of cartilage under deformation.
\end{abstract}

(c) 2014 Elsevier Ltd. All rights reserved.

\section{Introduction}

Most of the biomechanical studies consider that the temperature of the healthy knee cartilage is constant (Romanovsky, 2007; Tandon and Bali, 1997). However, given that cartilage is avascular and has viscous properties (Hayes and Mockros, 1971; Huang et al., 2003), a local temperature increase in this tissue could result from dissipation following a cyclic mechanical loading (Abdel-Sayed et al., 2014a, 2014b; Bali and Sharma, 2011). While this temperature increase could be moderate, its effect can be important on biochemical reactions. For example it has been shown that the rate of proteoglycan synthesis of chondrocytes increase with slight change in temperature (Brand et al., 1991; Castor and Yaron, 1976). Synthesis rates of hyaluronic acid by synovial cells changes as a function of temperature (Castor and Yaron, 1976). Furthermore, it has been reported that the destruction rate of articular cartilage collagen fibers by synovial collagenase is 4 times greater at $36^{\circ} \mathrm{C}$ than at $33{ }^{\circ} \mathrm{C}$ (Harris and McCroskery, 1974) and it affects the transport of molecules like polysaccharides and proteins in articular cartilage (Moeini et al., 2012).

An indirect link exists then between the dynamic loading of knee and the cartilage homeostasis through dissipative phenomenon

\footnotetext{
* Correspondence to: EPFL/STI/IBI/LBO, Station 19, 1015 Lausanne, Switzerland. Tel.: +4121693 8341; fax: +41216938660.

E-mail address: dominique.pioletti@epfl.ch (D.P. Pioletti).
}

suggesting a possible new mechanobiological pathway in cartilage (Abdel-Sayed et al., 2014a, 2014b).

Beside the dissipative properties of the cartilage, heat transfer is an important parameter in the local temperature regulation (Bali and Sharma, 2011; Martin et al., 2001; Tandon and Bali, 1997). In particular, synovial fluid flow would naturally play a central role in the heat transfer aspect. The performance of human articular joints is indeed strictly connected with the properties of the synovial fluid, which determines load transmission, lubrication, wear inhibition and protection of the articular cartilage from mechanical stresses during joint movements (Bali and Sharma, 2011; Hui et al., 2012). The lubricant properties of synovial fluid are essential in preventing the temperature increase due to friction (Bergmann et al., 2001a, 2001b; Sawyer et al., 2003; Tandon and Bali, 1997). However, how the synovial fluid flow affects the temperature increase due to the intrinsic viscous dissipation of the cartilage has not been described yet.

The goal of this study is to evaluate the impact of the synovial fluid flow on heat transfer in knee cartilage. We specifically focused on the heat generated due to viscous dissipation when the cartilage is deformed sinusoidally to mimic walking.

\section{Methods}

\subsection{Model of heat transfer in cartilage}

The theory of porous media for heat transfer in biological tissues was used in this study (Khaled and Vafaib, 2003). The problem was formulated as a two steps 
model. In the first step, the velocity field of the synovial fluid in the cartilage under deformation was determined in a poroelastic 3D finite element model. In the second step, the solution of the velocity field was introduced into the thermal diffusion equation. The thermal field within the porous cartilage was then determined.

Comsol Multiphysics 4.4 software (COMSOL Inc., Burlington, MA, USA) was used to develop the poroelastic, heat transfer model. The axisymmetric mode consisted of femoral and tibial cartilage layers each with the thickness of $4 \mathrm{~mm}$. As the contact area of cartilage $\left(1200 \mathrm{~mm}^{2}\right.$ ) comparing to its thickness (max $4 \mathrm{~mm}$ ) is large enough, to simplify the model the articulation shape of the cartilage was neglected (Liu et al., 2010). The cartilage was considered as a poroelastic material. As $80 \%$ percent of the cartilage is composed of fluid, the porosity was set accordingly to 0.8 (Hosoda et al., 2009). Both cartilage layers were attached to their corresponding femoral and tibial bones. Bone was modeled as rigid body. The synovial cavity was modeled as a reservoir for synovial fluid, which allowed the exchange of the fluid at each deformation cycle. The geometry was meshed with triangular elements (Fig. 1). The simulation consisted of two coupled multiphysics: poroelasticity and heat transfer interfaces.

Poroelasticity interface: calculation of the velocity field of the synovial fluid. The Biot's theory of poroelasticity was used, relating continuity equation for fluid flow in porous medium (1) and Navier's Eq. (2) (Biot, 1962):

$\rho_{l} S \frac{\partial p_{l}}{\partial t}+\nabla \times\left(\rho_{l} u\right)=-\rho_{l} \alpha_{B} \frac{\partial \epsilon_{v o l}}{\partial t}$

$-\nabla \boldsymbol{\sigma}=0$

where $u$ and $S$ are:

$u=-\frac{k}{\mu} \nabla p_{l}$

$S=\epsilon_{p} \chi_{l}+\frac{\left(\alpha_{B}-\epsilon_{p}\right)\left(1-\alpha_{B}\right)}{K}$

$\partial \varepsilon_{v o l} / \partial t$ is the rate of change in volumetric strain, $p_{l}$ is the pressure field, $\rho_{l}$ is the density of the fluid, and $\alpha_{B}$ is the Biot-Willis coefficient, which is considered equal to 1 in our situation. The material properties needed for the poroelastic model (Eqs. (1)-(4)) are listed in Table 1. The strain dependent permeability, $k$ was also implemented using the variable void ratio as (Mansour and Mow, 1976):

$k=k_{0}\left(\frac{1+e}{1+e_{0}}\right)^{M}, \quad e=\frac{\Theta_{l}}{\Theta_{p}}$

where $k_{0}$ is the initial permeability (equal to $10^{-14} \mathrm{~m}^{2}$ ), $e$ is void ratio (defined as the ratio of fluid volume fraction $\Theta_{l}$ to solid volume fraction $\Theta_{p}$ ), $e_{0}$ is the initial void ratio (equal to 4 ), and $M$ is the permeability coefficient, with the value 1.3 (Prendergast et al., 1996).

This model allows us to quantify the synovial fluid flow inside the cartilage when the cartilage is sinusoidally deformed. Both cartilage layers were deformed at a frequency of $1.5 \mathrm{~Hz}$ and a $15 \%$ amplitude to simulate gagging when the tibial bone is fully constrained (Liu et al., 2010). A zero pressure boundary condition $(p=0)$ was defined at the contact surface of the two cartilages to let the fluid flows in and out of the porous cartilage from the cartilage surface during deformation. At each time step, the pressure and velocity distribution were calculated.

A

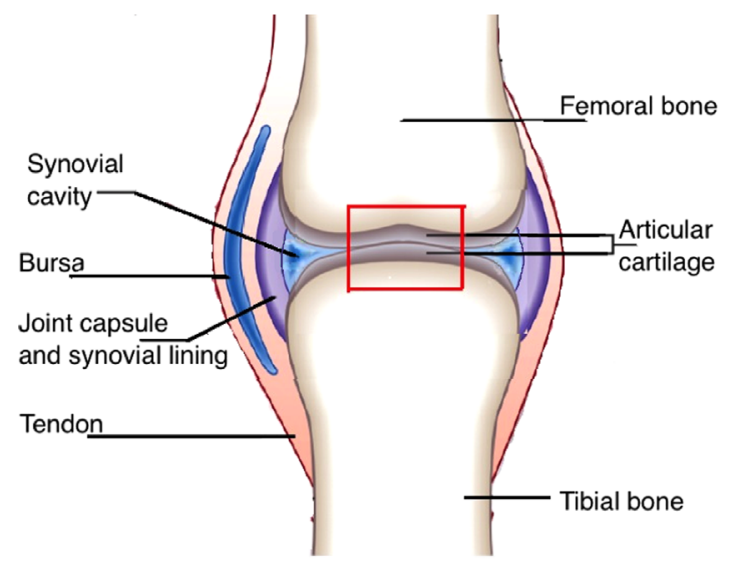

Heat transfer interface: calculation of the thermal field. The following form of the heat equation was used to model the heat transfer in cartilage (Incropera et al., 2011):

$\left(\rho C_{p}\right)_{e q} \frac{\partial T}{\partial t}+\rho_{l} C_{l} u \times \nabla T=\nabla \times\left(k_{e q} \nabla T\right)+\dot{Q}$

In this equation $u$ is the fluid velocity (introduced in Eq. (3)). At each time step the fluid velocity magnitude was solved from the poroelastic model and was used in Eq. (6) for calculating the temperature distribution. The equivalent conductivity of the solid-fluid system, $k_{e q}$ was related to the conductivity of the solid, $k_{p}$ and to the conductivity of the fluid, $k_{l}$ by $k_{e q}=\Theta_{p} k_{p}+\Theta_{l} k_{l}$. The equivalent volumetric heat capacity of the solid-fluid system was related to the heat capacity of the solid $C_{p}$ and to the heat capacity of the fluid $C_{l}$ by $\left(\rho C_{p}\right)_{e q}=\Theta_{p} \rho_{p} C_{p}+\Theta_{l} \rho_{l} C_{l}$. The heat capacity and conductivity of the cartilage as well as of the synovial fluid were measured experimentally (see below).

In Eq. (6), $Q$ is the heat source, which in our model corresponded to the cartilage viscous dissipation. The amount of cartilage dissipation was measured experimentally. To evaluate other possible heat transfer phenomena between the cartilage and the surrounding area, we considered a heat transfer boundary condition at the free boundary of the model by introducing a general heat transfer coefficient $(\lambda)$ experimentally determined.

\subsection{Experimental determination of the model parameters}

Dissipation of cartilage: we applied cyclic compression on human cartilage samples $(n=6)$ at $1.5 \mathrm{~Hz}$ and $15 \%$ strain amplitude. The dissipation power was calculated from the load-displacement graphs as the integral of the area enclosed by the hysteresis curve. The detailed procedure is explained in a previous study (Abdel-Sayed et al., 2014a, 2014b).

Heat capacity of cartilage and synovial fluid: heat capacity of human cartilage samples $(n=6)$ and synovial fluid $(n=3)$ were determined using a general published differential scanning calorimeter (DSC) method (McHugh et al., 2010). Briefly, the DSC heat flow signal from the sample was recorded between $10{ }^{\circ} \mathrm{C}$ and $60{ }^{\circ} \mathrm{C}$ with a heat rate of $10^{\circ} \mathrm{C} / \mathrm{min}$ (MDS-Q100, TA Instruments, New Castle, DE, USA). The DSC signal was compared to the DSC signal of sapphire calibration standard with a known specific heat. Both curves were corrected by a base line correction experiment.

Thermal conductivity of cartilage: as large pieces of cartilage samples were necessary to evaluate their thermal conductivity, for this measurement, cartilage samples $(n=6)$ were punched $(20 \mathrm{~mm}$ diameter) from bovine femoral head. Hot Disk Thermal Constants Analyzer (TPS-500, Hot Disk, Gothenburg, Sweden) was used to measure the heat conductivity of cartilage. The method is based on the

Table 1

Material properties used in the model (Barker and Seedhom, 2001; Mow et al., 1984).

\begin{tabular}{ll}
\hline Bulk modulus of cartilage, $K$ & $0.36 \mathrm{MPa}$ \\
\hline Viscosity of synovial fluid, $\mu$ & $0.6 \mathrm{~Pa} \mathrm{~s}$ \\
Porosity of cartilage, $\epsilon_{p}$ & 0.8 \\
Compressibility of synovial fluid, $\chi_{l}$ & $1 \mathrm{e}-101 / \mathrm{Pa}$ \\
Density of fluid, $\rho_{l}$ & $1000 \mathrm{~kg} / \mathrm{m}^{3}$ \\
\hline
\end{tabular}

B

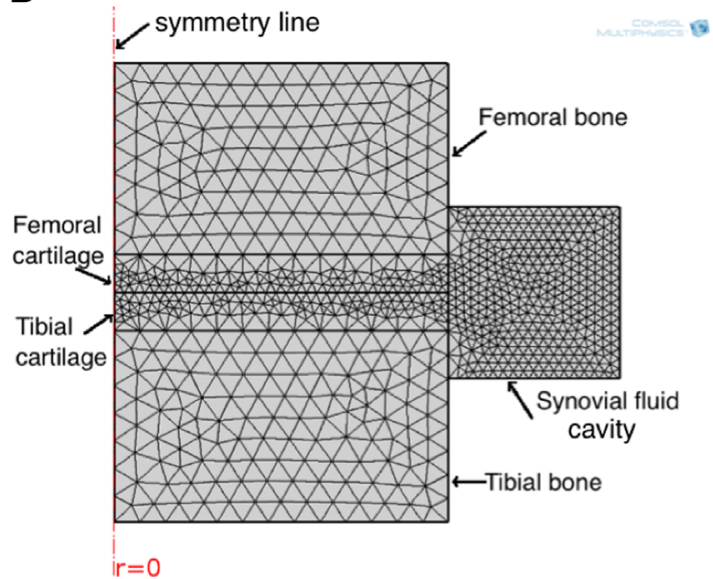

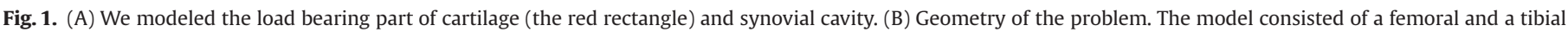

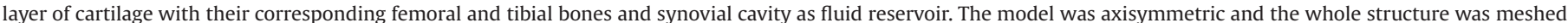
with triangular elements. (For interpretation of the references to color in this figure legend, the reader is referred to the web version of this article.) 
use of a transiently heated plane sensor. The Hot Disk sensor consists of an electrically conducting pattern in the shape of a double spiral, which has been etched out of a thin metal (Nickel) foil. This spiral is sandwiched between two thin sheets of an insulating material. The Hot Disk sensor is used both as a heat source and as a dynamic temperature sensor. The test was also performed on $3 \mathrm{~mL}$ of bovine synovial fluid in a $5 \mathrm{~mL}$ tube by sinking the Hot Disk sensor directly in the liquid.

Heat transfer coefficient of cartilage: the heat transfer coefficient $(\lambda)$ was obtained from a Proton Resonance Frequency Shift (PRFS)-based MR thermometry used to monitor the temperature drop of the cartilage when the knee of 4 volunteers was immobilized after a 20 min joint activity (Abdel-Sayed et al., 2014a, 2014b). We calculated the heat transfer coefficient $(\lambda)$ from the following equation:

$a=\frac{\lambda S}{m c}$

$a$ is the time constant derived form the curve fitting to the temperature drop curve of the cartilage following the 20 min joint activity and was detected by MR thermometry method (Abdel-Sayed et al., 2014a, 2014b), $S$ is the surface of each cartilage element in MRI data, $m$ is the mass of each element and $c$ is the heat capacity of the cartilage. Since the heat transfer coefficient $(\lambda)$ was calculated when the knee was at rest, meaning that no dynamic load was applied on cartilage to cause fluid flow, it includes all heat transfer mechanisms at the knee cartilage except the effect of synovial fluid flow.

\section{Results}

\subsection{Experimental determination of the model parameters}

Dissipation of cartilage: the dynamic compression test on cartilage induced a dissipation equal to $0.025 \pm 0.009 \mathrm{~mW} / \mathrm{mm}^{3}$. This value was considered as an internal heat source over the whole cartilage geometry.

Heat capacity and thermal conductivity of cartilage and synovial fluid: the obtained experimental values are shown in Table 2. These values are used to for the parameters appearing in Eq. (6) describing the heat transfer model of the cartilage porous media.

Heat transfer coefficient of cartilage: the results of MR thermometry on 4 volunteers give the value of $\lambda=0.77 \pm 0.32 \mathrm{~J} / \mathrm{m}^{2} \mathrm{~s}$. The

Table 2

DSC and TPS experimentally obtained values for cartilage and synovial fluid.

$\begin{array}{ll}\text { Heat capacity of cartilage, } C_{p} & 3200 \pm 130 \mathrm{~J} / \mathrm{kg} \mathrm{K} \\ \text { Heat capacity of synovial fluid, } C_{l} & 3900 \pm 95 \mathrm{~J} / \mathrm{kg} \mathrm{K} \\ \text { Conductivity of cartilage, } k_{p} & 0.21 \pm 0.06 \mathrm{~W} / \mathrm{m} \mathrm{K} \\ \text { Conductivity of synovial fluid, } k_{l} & 0.62 \pm 0.11 \mathrm{~W} / \mathrm{m} \mathrm{K}\end{array}$

value of this heat transfer coefficient was considered at all open boundaries of the cartilage model.
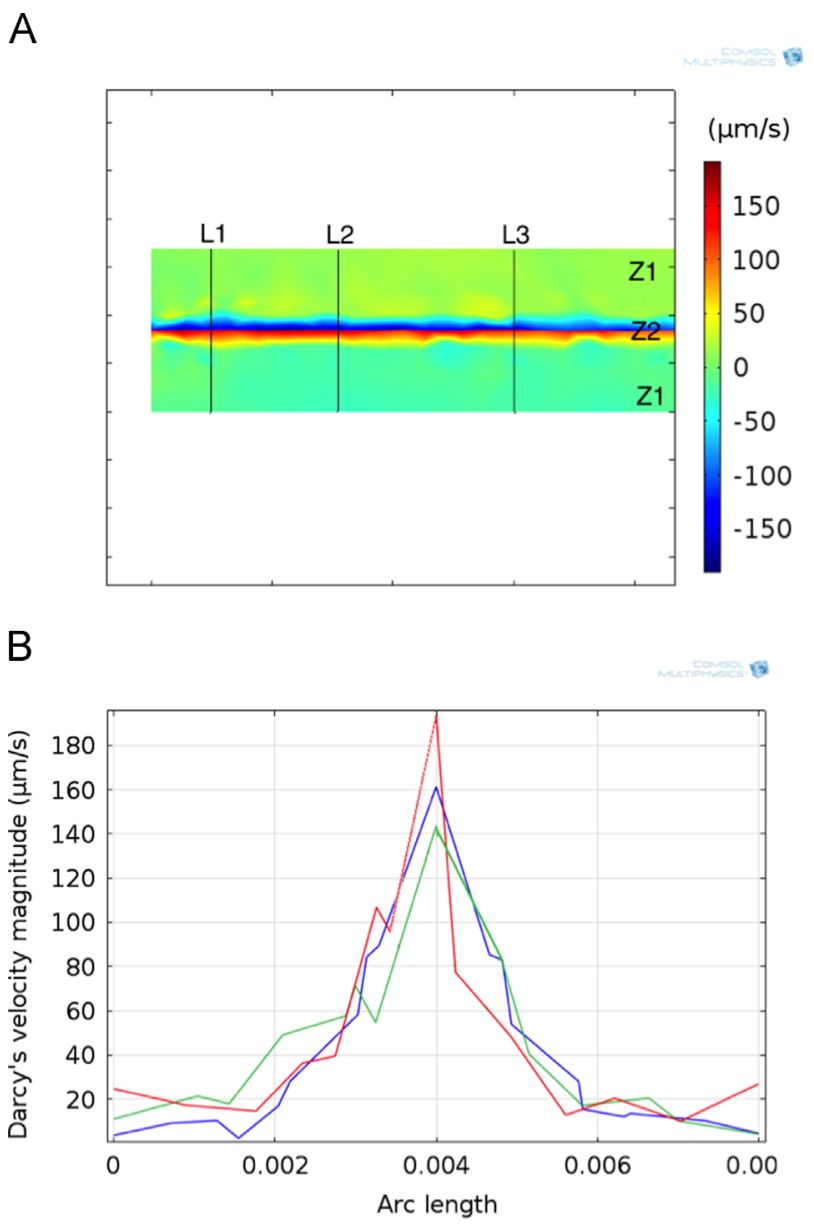

Fig. 3. Maximum velocity field in cartilage (A) and velocity magnitude in 3 areas, green: along the line L1, blue along the line L2 and red along the line L3 (B). (For interpretation of the references to color in this figure legend, the reader is referred to the web version of this article.)

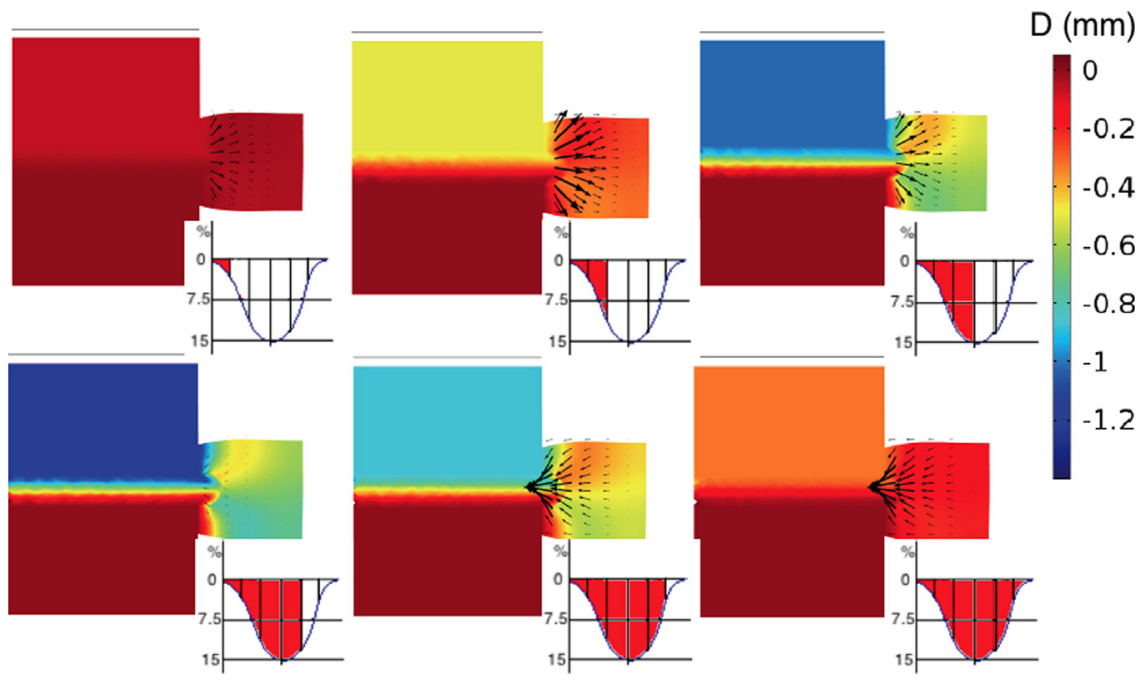

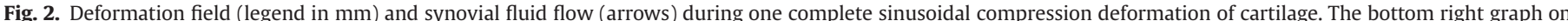
each figure shows the stage of deformation during one sinusoidal compression. 
A

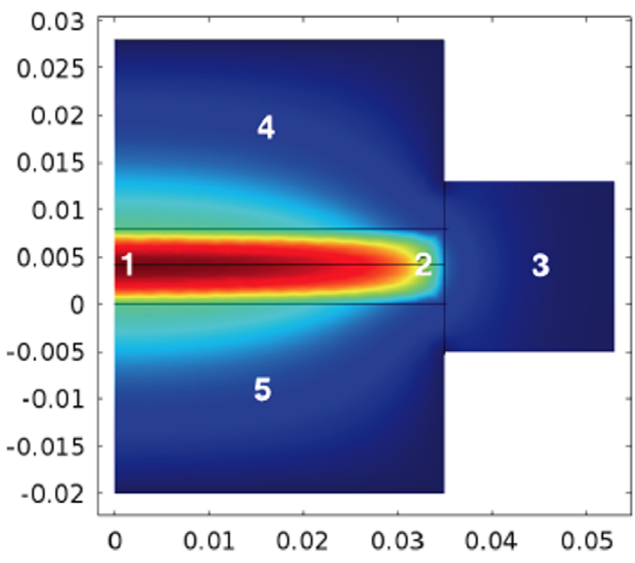

B

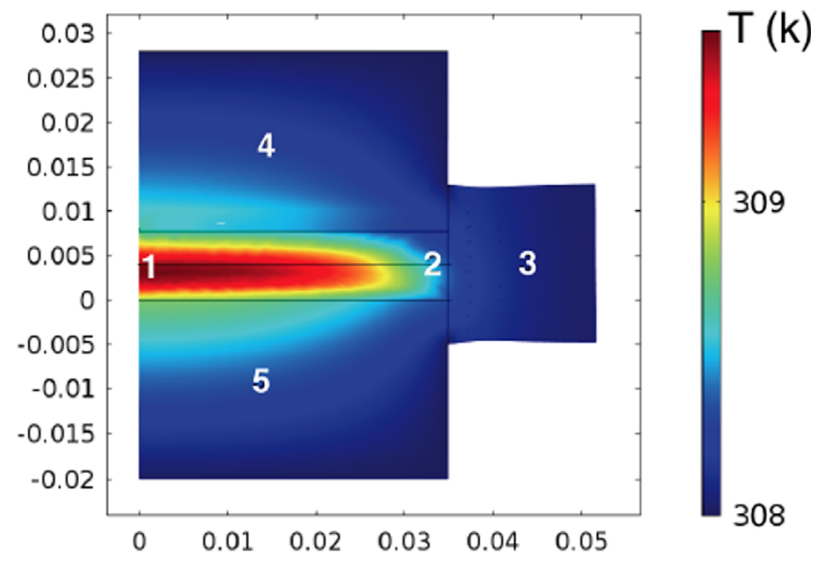

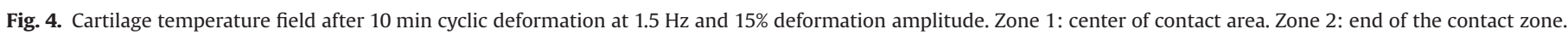
Zone 3: synovial fluid reservoir. Zones 4 and 5: femoral and tibial bones.

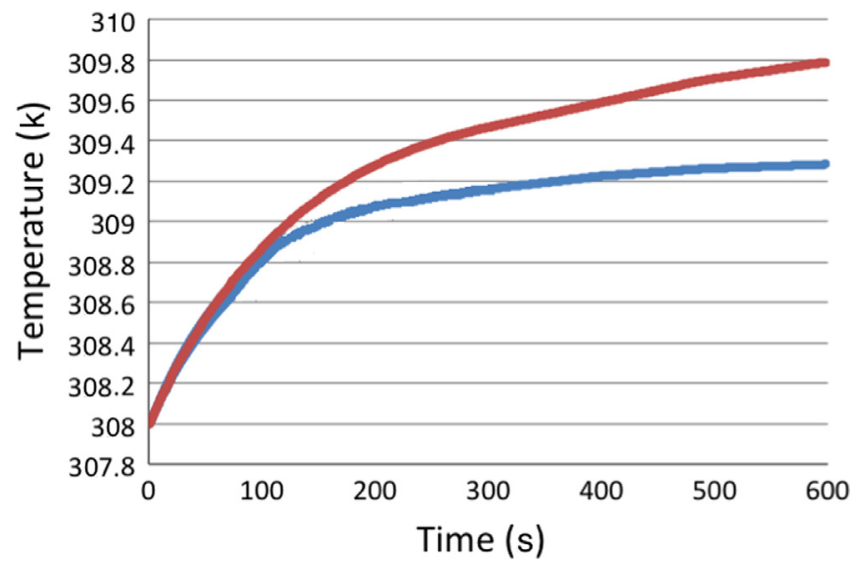

Fig. 5. Mean temperature in the middle of the cartilage contact zone over time with (blue line) and without (red line) synovial fluid flow. (For interpretation of the references to color in this figure legend, the reader is referred to the web version of this article.)

\subsection{Model of heat transfer in cartilage}

Velocity field of the synovial fluid: to evaluate the pertinence of the calculated synovial fluid flow during cyclic loading in our numerical model, we first looked at the cartilage deformation and fluid flow. Fig. 2 shows the cartilage deformation between two layers of tibial and femoral bone during one complete cycle of loading and unloading. The black arrows show the fluid velocity in and out of the porous cartilages during exudation and imbibition. During the cartilage compression corresponding to stance phase of gait cycle, fluid is squeezed out of the cartilage to the synovial fluid reservoir cavity. Conversely, during unloading corresponding to the swing phase of gait cycle, cartilage sucks back the fluid. Fig. 3 provides a closer look at the maximum fluid velocity in cartilage during cyclic compression. The fluid velocity at the area near the cartilage surface (Zone Z2, Fig. 3A) is more that 10 times higher than the velocity in deep zones (Zones Z1, Fig. 3A).

Effect of synovial fluid flow in the regulation of cartilage temperature. Fig. 4 shows the spatial distribution of knee cartilage temperature due to its viscous dissipation after $10 \mathrm{~min}$ cyclic loading at $1.5 \mathrm{~Hz}$. Results are presented with or without considering the synovial fluid flow. The flow of fluid has not a significant effect on temperature of cartilage at the contact area. An effect can be observed in the area near the synovial fluid cavity (Zone 2, Fig. 4) where the fluid can be exchanged faster. Fig. 5 represents the mean temperature increase of cartilage in the center of the cartilage contact surface (Zone 1, in Fig. 4). The model showed that the dissipation could increase the temperature of knee cartilage up to $1.2^{\circ} \mathrm{C}$ after $10 \mathrm{~min}$ loading. This temperature increase without fluid flow was about $1.8^{\circ} \mathrm{C}$.

\section{Discussion}

In this study we evaluated the effect of the cartilage dissipation on its temperature distribution and we quantified the importance of the synovial fluid flow in this process.

An original finding was to show that the experimentally determined values of the heat capacity and conductivity of the synovial fluid were much higher than the corresponding values for the cartilage tissue. This suggests that the synovial fluid would play an important role in the temperature regulation of the knee cartilage during dynamic loading. However, based on the developed numerical poroelastic model coupled to a thermal diffusion model, we observed that the synovial fluid flow could only partially affect the temperature increase in the cartilage submitted to a dynamic loading. Indeed, a significant temperature increase in the cartilage was observed even when considering the synovial fluid flow. This result allows us to confirm the importance of the dissipation on cartilage temperature increase observed in a previous study where the contribution of the fluid flow could not be included (Abdel-Sayed et al., 2014a, 2014b).

One possible reason to explain the inability of the synovial fluid flow to maintain a constant temperature in the cartilage during its dynamical loading may be related to the cartilage low permeability value $\left(10^{-14} \mathrm{~m}^{2}\right.$ e.g. (Prendergast et al., 1996)). The low permeability of the cartilage is central to provide enough mechanical properties and shock absorbance (Mow et al., 1984). It also provides a moderate fluid flow to the deep zone of cartilage, enough to transport nutrient and waste in this tissue, where there is no vascularization (O'Hara et al., 1990). At the frequency of activities like walking or running, it seems that such a low permeability does not let the synovial fluid to travel fast enough through the deep zone of cartilage to allow an effective heat exchange at every deformation cycle. Fig. 3 supports this suggestion as it is reported that the fluid velocity at the area near the cartilage surface is more that 10 times higher than the velocity in deep zones. It may be worth mentioning that no significant differences were found in the model if 1 or $5 \mathrm{~Hz}$ instead of $1.5 \mathrm{~Hz}$ were used (data not shown). Within this context, the role of the synovial fluid flow and convectional heat transfer seems not to be significant. Therefore, the heat produced due to viscous dissipation at the deep zone of the cartilage can only be transferred through the conductivity of cartilage. On the other hand, the fast 
exchange of synovial fluid at the thin layer of the cartilage surface observed in our study confirms the role of the synovial fluid for joint lubrication and for removing heat produced by cartilage surface friction (Gleghorn et al., 2009; Schmidt et al., 2007).

In this study we simplified our model considering only the loadbearing part of cartilage where dissipation occurs. An underestimation of heat transfer phenomena may then result by not considering heat produced by muscle activities around the joint or heat transfer through the skin. To overcome this limitation, a general heat transfer coefficient $(\lambda)$ has been introduced at the boundaries allowing to take into account other heat phenomena not related to dissipation or fluid flow in our model. Another simplification of our description was to model the tibial and femoral bones as rigid bodies, while bone is usually considered as a poroelastic material. However, the permeability of cartilage is much higher than the permeability of bone (Mow et al., 1984), so the effects of bone poroelasticity on the synovial fluid flow could be neglected.

The results of the developed model can be compared to other previously published models either for the mechanical or for the thermal aspects. Regarding the mechanical aspects, our model shows similar fluid flow profiles compared to previous studies (Barker and Seedhom, 2001; Liu et al., 2010; Mow et al., 1984). However, our model has the advantage to show the velocity magnitude in different zone of cartilage, which to our knowledge has not been yet presented. Based on the calculated velocity at the surface or deep zones of cartilage, we could propose an explanation to better understand the reason why synovial fluid flow had not a significant role in regulating the temperature increase due to dissipation while having a central role in case of frictional heat. Regarding the thermal aspects, in vivo direct measurement of intra-articular knee temperature has shown a temperature increase up to $4{ }^{\circ} \mathrm{C}$ after an hour of activity (Becher et al., 2008; Trobec et al., 2008). However, in these studies it was not possible to access the cartilage temperature, only the intra-articular knee temperature could be measured. Furthermore, in most of the existing numerical/mathematical models, viscous dissipation has not been considered as a heat source, and considerations were given only to friction between the surfaces (Romanovsky, 2007; Tandon and Bali, 1997). Bali and coworkers have reported in their poroelastic model the temperature increase of $1.5{ }^{\circ} \mathrm{C}$ subsequent to cyclic deformation (Bali and Sharma, 2011; Tandon and Bali, 1997). Nevertheless they did not include the effects of synovial fluid flow in the tissue and related gradients of temperature.

In conclusion, we showed that cartilage viscous dissipation is an important source of heat that can locally increase the cartilage temperature. The synovial fluid cannot significantly prevent this temperature increase when the cartilage is mechanically loaded. As previously mentioned, the resulting increase of temperature may induce profound effects on the metabolism of cartilage.

\section{Conflict of interest statement}

None of the author has any conflict of interest.

\section{Acknowledgments}

This work was supported by the Swiss National Science Foundation (\#406240_126070, PNR 62 program).

\section{References}

Abdel-Sayed, P., Darwiche, S.E., Kettenberger, U., Pioletti, D.P., 2014a. The role of energy dissipation of polymeric scaffolds in the mechanobiological modulation of chondrogenic expression. Biomaterials 35, 1890-1897.
Abdel-Sayed, P., Moghadam, M.N., Salomir, R., Tchernin, D., Pioletti, D.P., 2014b. Intrinsic viscoelasticity increases temperature in knee cartilage under physiological loading. J. Mech. Behav. Biomed. Mater. 30, 123-130.

Bali, R., Sharma, S., 2011. A model for intra-articular heat exchange in a knee joint. Tribol. Lett. 41, 379-386.

Barker, M., Seedhom, B., 2001. The relationship of the compressive modulus of articular cartilage with its deformation response to cyclic loading: does cartilage optimize its modulus so as to minimize the strains arising in it due to the prevalent loading regime? Rheumatology 40, 274-284.

Becher, C., Springer, J., Feil, S., Cerulli, G., Paessler, H., 2008. Intra-articular temperatures of the knee in sports, an in-vivo study of jogging and alpine skiing. BMC Musculoskelet. Disord. 9, 46-52.

Bergmann, G., Graichen, F., Rohlmann, A., Verdonschot, N., Van Lenthe, G., 2001a. Frictional heating of total hip implants. Part 2: Finite element study. J. Biomech. 34, 429-435.

Bergmann, G., Graichen, F., Rohlmann, A., Verdonschot, N., van Lenthe, G.H., 2001b. Frictional heating of total hip implants, Part 1: Measurements in patients. J. Biomech. 34, 421-428.

Biot, M.A., 1962. Mechanics of deformation and acoustic propagation in porous media. J. Appl. Phys. 33, 1482-1498.

Brand, H.S., De Koning, M.H.M.T., Van Kampen, G.P.J., Van Der Korst, J.K., 1991. Effect of temperature on the metabolism of proteoglycans in explants of bovine articular cartilage. Connect. Tissue Res. 26, 87-100.

Castor, C.W., Yaron, M., 1976. Connective tissue activation: VIII. The effects of temperature studied in vitro. Arch. Phys. Med. Rehabil. 57, 5-9.

Gleghorn, J.P., Jones, A.R., Flannery, C.R., Bonassar, L.J., 2009. Boundary mode lubrication of articular cartilage by recombinant human lubricin. J. Orthop. Res.: official publication of the Orthopaedic Research Society 27, 771-777.

Harris, E.D., McCroskery, P.A., 1974. The influence of temperature and fibril stability on degradation of cartilage collagen by rheumatoid synovial collagenase. N. Engl. J. Med. 290, 1-6.

Hayes, W., Mockros, L., 1971. Viscoelastic properties of human articular cartilage. J. Appl. Physiol. 31, 562-568.

Hosoda, N., Sakai, N., Sawae, Y., Murakami, T., 2009. Finite element analysis of articular cartilage model considering the configuration and biphasic property of the tissue. In: Lim, C.T., Goh, J.C.H. (Eds.), 13th International Conference on Biomedical Engineering. Springer, Berlin Heidelberg, pp. 1883-1887.

Huang, C.-Y., Soltz, M.A., Kopacz, M., Mow, V.C., Ateshian, G.A., 2003. Experimental verification of the roles of intrinsic matrix viscoelasticity and tension-compression nonlinearity in the biphasic response of cartilage. J. Biomech. Eng. 125, 84-93.

Hui, A.Y., McCarty, W.J., Masuda, K., Firestein, G.S., Sah, R.L., 2012. A systems biology approach to synovial joint lubrication in health, injury, and disease. Wiley Interdiscip. Rev. Syst. Biol. Med. 4, 15-37.

Incropera, F.P., Bergman, T.L., Lavine, A.S., DeWitt, D.P., 2011. Fundamentals of Heat and Mass Transfer. Wiley.

Khaled, A.-R.A., Vafaib, K., 2003. The role of porous media in modeling flow and heat transfer in biological tissues. Int. J. Heat Mass Transfer 46, 4989-5003.

Liu, F., Kozanek, M., Hosseini, A., Van de Velde, S.K., Gill, T.J., Rubash, H.E., Li, G. 2010. In vivo tibiofemoral cartilage deformation during the stance phase of gait J. Biomech. 43, 658-665.

Martin, S.S., Spindler, K.P., Tarter, J.W., Detwiler, K., Petersen, H.A., 2001. Cryotherapy: an effective modality for decreasing intraarticular temperature after knee arthroscopy. Am. J. Sports Med. 29, 288-291.

McHugh, J., Fideu, P., Herrmann, A., Stark, W., 2010. Determination and review of specific heat capacity measurements during isothermal cure of an epoxy using TM-DSC and standard DSC techniques. Polym. Test. 29, 759-765.

Moeini, M., Lee, K.-B., Quinn, T.M., 2012. Temperature affects transport of polysaccharides and proteins in articular cartilage explants. J. Biomech. 45, 1916-1923.

Mansour, J.M., Mow, V.C., 1976. The permeability of articular cartilage under compressive strain and at high pressures. J. Bone Joint Surg. Am. 58, 509-516.

Mow, V.C., Holmes, M.H., Michael Lai, W., 1984. Fluid transport and mechanical properties of articular cartilage: a review. J. Biomech. 17, 377-394.

O’Hara, B., Urban, J., Maroudas, A., 1990. Influence of cyclic loading on the nutrition of articular cartilage. Ann. Rheum. Dis, 49, 536-539.

Prendergast, P., Van Driel, W., Kuiper, J., 1996. A comparison of finite element codes for the solution of biphasic poroelastic problems. Proc. Inst. Mech. Eng. Part H J. Eng. Med. 210, 131-136.

Romanovsky, A.A., 2007. Thermoregulation: some concepts have changed. Functional architecture of the thermoregulatory system. Am. J. Physiol. Regul. Integr. Comp. Physiol. 292, R37-R46.

Sawyer, W., Hamilton, M., Fregly, B., Banks, S., 2003. Temperature modeling in a total knee joint replacement using patient-specific kinematics. Tribol. Lett. 15 343-351.

Schmidt, T.A., Gastelum, N.S., Nguyen, Q.T., Schumacher, B.L., Sah, R.L., 2007 Boundary lubrication of articular cartilage: role of synovial fluid constituents. Arthritis Rheum. 56, 882-891.

Tandon, P.N., Bali, R., 1997. A study on temperature regulation in synovialjoints. Tribol. Lett. 3, 209-213.

Trobec, R., Sterk, M., AlMawed, S., Veselko, M, 2008. Computer simulation of topical knee cooling. Comput. Biol. Med. 38, 1076-1083. 